\title{
Spatial Distribution of Motor Unit Fibers in the Cat Soleus and Tibialis Anterior Muscles: Local Interactions
}

\author{
Sue C. Bodine, Alan Garfinkel, Roland R. Roy, and V. Reggie Edgerton \\ Brain Research Institute and Department of Kinesiology, University of California, Los Angeles, California 90024
}

\begin{abstract}
The spatial distribution of muscle fibers belonging to a motor unit was studied in the soleus and tibialis anterior muscles of adult cats. Motor unit fibers were depleted of their glycogen through repetitive stimulation of the motoneuron or of the functionally isolated motor axon. Subsequently, the position of depleted muscle fibers was mapped on serial cross sections taken along the length of the muscle. A subset of fibers was selected from the cross section containing the largest number of motor unit fibers and $\mathbf{4}$ spatial analyses were performed. These analyses were designed to determine whether the muscle fibers belonging to a single unit were distributed in a random manner. To test whether the actual distribution was other than random, Monte Carlo techniques were used to simulate the random innervation of a muscle. From these simulations, a test statistic was calculated for comparison with the observed data. Adjacency and nearest-neighbor analyses revealed no tendency for grouping or dispersion of fibers belonging to a motor unit. However, measurement of the distances between all motor unit fibers revealed a greater tendency for grouping than spreading, suggesting the existence of some mechanism that restricts the absolute distribution and territory of a motor unit.
\end{abstract}

The processes responsible for the formation of highly ordered and specific connections are a central issue in developmental neurobiology. In many systems the initial pattern of innervation is diffuse, becoming highly organized at later stages (for review, see Purves and Lichtman, 1984). In muscle, motoneurons are ablc to find thcir appropriate targets with remarkable accuracy (Landmesser, 1980; Bennett, 1983); however, the degree to which a motoneuron selectively innervates muscle fibers with similar properties is unknown. Generally, it has been assumed that the connections between motor axons and the muscle fibers they innervate show little organization beyond selectivity for a given muscle. However, recent evidence suggests that the specification may be ordered to a finer degree than just motoneuron pool to muscle. Glycogen depletion studies in several mammalian hindlimb muscles have revealed that a muscle is often subdivided into compartments that are preferentially innervated by one

\footnotetext{
Received July 7, 1987; revised Oct. 15, 1987; accepted Oct. 20, 1987.

This work was supported by National Institute of Neurological and Communicative Disorders and Stroke Grant NS-16333. We wish to thank Drs. Tim C.

Cope and John A. Hodgson for their contributions to the collection of this data.

This paper was adapted from a thesis written by S.C.B. in partial fulfillment of

the requirements for the Ph.D. degree at the University of California, Los Angeles.

Correspondence should be addressed to Sue Bodine-Fowler, Ph.D., Department

of Kinesiology, 2854 Slichter Hall, UCLA, 405 Hilgard Ave., Los Angeles, CA 90024-1568.

Copyright (C) 1988 Society for Neuroscience $0270-6474 / 88 / 062142-11 \$ 02.00 / 0$
}

muscle nerve branch (English and Weeks, 1984; Iliya and Dum, 1984). In the cat lateral gastrocnemius, fibers belonging to a single motor unit are distributed only within the boundaries of one compartment (English and Weeks, 1984). Further studies have shown that a muscle receives topographically organized projections from the motor pool (Donselaar et al., 1985; Weeks and English, 1985; Laskowski and Sanes, 1987) and that a motor axon may selectively innervate muscle fibers of a specific type (McLennan, 1983; Thompson et al., 1984). These findings suggest that the process of innervation is not random and that a given motoneuron does not have an equal probability of innervating all fibers within a muscle. In order to determine whether further organization exists at the level of individual fibers, the spatial distribution of muscle fibers belonging to a single motor unit was analyzed in this study.

The spatial distribution of single motor unit fibers in normal muscle must reflect the sum total of those factors that influence the formation and elimination of synapses. For example, if the fibers belonging to a motor unit are separated by a distance greater than would be expected from a random distribution or are rarely in contact with one another, then presumably some active process(es) must be influencing the formation of nervemuscle connections. Visualization of the fibers belonging to a motor unit has been made possible through glycogen depletion techniques (Edstrom and Kugelberg, 1968). On the basis of visual impressions from motor units that have been depleted of glycogen, the normal pattern of motor unit fibers has been characterized by such terms as "random," "scattered," "irregularly spaced," and "uniformly dispersed" (Edstrom and Kugelberg, 1968; Brandstater and Lambert, 1973; Kugelberg, 1973). In several studies, however, the spatial distribution of motor unit fibers has been measured more directly by counting the number of incidences in which fibers of a single motor unit are adjacent to one another. Brandstater and Lambert (1973) reported that the number of adjacencies among motor unit fibers was not different from random, whilc Willison (1980) claimed to find evidence supporting nonrandom innervation, in that fewer adjacencies were found among motor unit fibers than would be expected from a random distribution. Other than these studies, we are unaware of any published reports that have attempted to quantify the spatial distribution of motor unit fibers in identified units. In humans, the distribution of motor unit fibers has been modeled using data obtained from the fiberdensity technique (Buchthal et al., 1957) of single-fiber electromyography and has been described as both uniform (Gath and Shenhav, 1985) and "quasi-Gaussian" (Miller-Larsson, 1980).

The present study was designed to test the hypothesis that the muscle fibers innervated by a single motoneuron are distributed in a random manner. To achieve this, the actual dis- 
tribution of motor unit fibers was determined and compared to what would be expected from a purely random process based on Monte Carlo simulation techniques. Some factors that could influence the spatial pattern of motor unit fibers range from cellto-cell contact or cell surface phenomena to limitations in the intramuscular branching of axons. This paper presents data from 4 analyses designed to investigate potential local or short-range interactions that may occur between motor unit fibers. Preliminary results have been reported elsewhere (Garfinkel et al., 1986).

\section{Materials and Methods}

The spatial distribution of fibers belonging to a motor unit was analyzed in 3 soleus (SOL) and 4 tibialis anterior (TA) units in 7 adult cats. Motor units were visualized using glycogen depletion techniques (Edstrom and Kugelberg, 1968), and the fibers belonging to the unit were identified on serial cross sections of the muscle. The distribution of motor unit fibers was subsequently analyzed using software developed for the microcomputer.

\section{Motor unit identification}

Experiments were performed under pentobarbital anesthesia, $35 \mathrm{mg} /$ $\mathrm{kg}$, i.p., supplemented intravenously as needed to keep withdrawal and eye-blink responses suppressed. The spinal cord was exposed from level L5 to S1 and the muscles of the tail, hip, thigh, and lower leg were denervated, with the exception of the TA or SOL, which was isolated from surrounding tissues, with care taken to preserve the blood supply to the muscle. In each TA muscle, one motor unit was characterized physiologically and glycogen depleted by stimulating its functionally isolated axon. Criteria for functional isolation of TA motor units were elicitation of (1) an all-or-none twitch response following filament stimulation at voltages ranging from threshold to $10 \times$ threshold, (2) a corresponding all-or-none EMG record from the muscle demonstrating a consistent waveform, and (3) an all-or-none action potential in the root filament following antidromic stimulation of the muscle nerve. Soleus motor units were isolated and stimulated using intracellular techniques, as described by Cope et al. (1986). Penetration of a SOL motoneuron was confirmed by (1) the occurrence of an antidromic action potential upon stimulation of the nerve to the muscle, and (2) the presence of an all-or-none twitch and EMG potential in the SOL upon stimulation of the motoneuron.

Glycogen depletion of the motor unit was achieved through repetitive stimulation of the muscle fibers, using a stimulus paradigm that optimized glycogen utilization and minimized neuromuscular junction failure (see Cope et al., 1986 and Bodine et al., 1987). Briefly, stimulation consisted of trains of 50-300 msec duration repeated at a rate of 2-5/ sec and a frequency of 40-75 Hz. A typical stimulation regime consisted of trains of $100 \mathrm{msec}$ duration repeated at a rate of $5 / \mathrm{sec}$ and a frequency of $75 \mathrm{~Hz}$. The amplitude of the EMG output of the unit was monitored throughout the stimulation period and used as an index to measure the functioning of the neuromuscular junction. In the SOL, glycogen depletion was facilitated by occlusion of the femoral artery. Upon cessation of stimulation, the musclc was exciscd, weighed, cut into blocks, and rapidly frozen in isopentane cooled to $-160^{\circ} \mathrm{C}$ with liquid nitrogen.

Motor units were classified physiologically as fast fatigable (FF), fast fatigue-intermediate (FI), fast fatigue-resistant (FR), or slow fatigueresistant (S), based on contraction time, fatigue index, maximum tension, and the presence or absence of sag during an unfused tetanus with an interpulse interval equal to 1.2 times contraction time (Cope et al., 1986; Bodine et al., 1987). In addition, serial cross sections stained histochemically for demonstration of myosin ATPase with alkaline preincubation (Nwoye et al., 1982) were used to classify muscle fibers as fast (darkly stained) or slow (lightly stained).

\section{Histological mapping of motor units}

Muscle fibers belonging to a stimulated motor unit were identified by their low level of glycogen. To assess the glycogen content of fibers, cross sections $(20 \mu \mathrm{m})$ were cut from the experimental muscle and stained using the periodic acid-Schiff reaction (Pearse, 1961). Using an image-processing computer system (Castleman et al., 1984; Martin et al., 1985), images of cross sections stained for glycogen were stored on

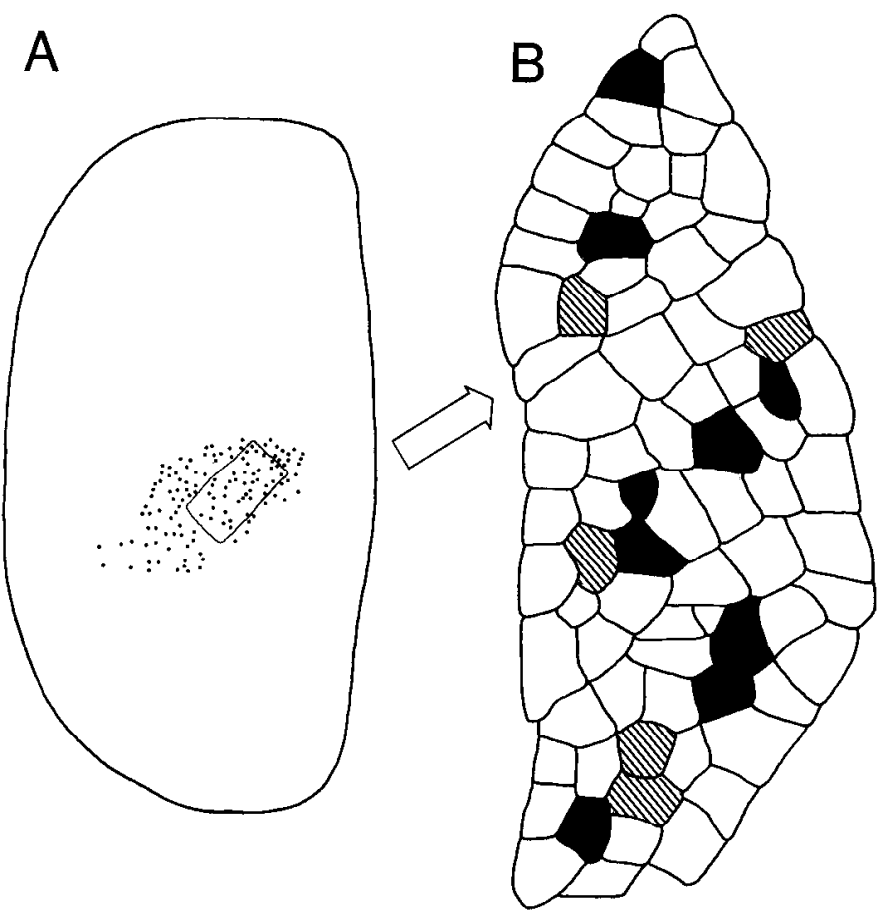

Figure 1. A, Distribution of depleted muscle fibers (solid circles) belonging to a single motor unit within a single muscle cross section. The outlined region represents that area within the motor unit territory that was selected for analysis. $B$, Schematic representation of a single fascicle within the selected area of the motor unit. All fibers within the selected area were outlined using an image-processing system and classified as being depleted or not depleted on the basis of periodic acid-Schiff staining, and as slow or fast on the basis of myosin ATPase (alkaline preincubation) staining. Muscle fibers in the fascicle are identified as depleted motor unit fibers (striped), slow, not-depleted fibers (solid), or fast, notdepleted fibers (open).

magnetic tape as gray-level pictures. From these pictures, muscle fibers were outlined manually, using a cursor, and measurements of the optical density of glycogen staining and cross sectional area were calculated. The position of glycogen- depeleted muscle fibers was mapped on serial cross sections taken along the length of the muscle and stained for periodic acid-Schiff. Using the cross section that contained the greatest number of motor unit fibers, the territory of the motor unit was defined as a convex polygon with borders defined by the most peripheral motor unit fibers. The spatial analyses were performed on a subset of fibers selected from within the motor unit territory and had an even density of motor unit fibers, as judged by visual inspection (Fig. 1A). Within the selected area, all fibers were outlined and identified as (1) depleted or not depleted, based on their optical density for glycogen staining, and (2) fast or slow, based on their myosin ATPase staining (Fig. 1B). In addition, each fiber was assigned a number, an $x, y$ coordinate corresponding to its centroid, and a cross-sectional area. Selected physiological properties of the 7 units and the fiber type compositions of the sample areas chosen for analysis are given in Table 1.

\section{Adjacency measurements}

Chi-square. A chi-square analysis was done using a $2 \times 2$ contingency table to test the hypothesis that the property of being or not being a motor unit fiber was distributed independently of whether there was an adjacency to a motor unit fiber. Two fibers were considered to be adjacent when their fiber boundaries were in contact. Each fiber in the sample was classified as either belonging (depleted) or not belonging (not depleted) to the stimulated motor unit and as being either adjacent or not adjacent to a motor unit fiber (Fig. $2 A$ ). The expected frequencies of each category in the $2 \times 2$ contingency table shown in Figure $2 A$ were derived by calculating the product of the row and column totals of that category and dividing this product by the total number of ob- 
Table 1. Physiological properties of motor units and fiber composition of sample area

\begin{tabular}{|c|c|c|c|c|c|c|c|c|c|c|}
\hline \multirow[b]{2}{*}{$\begin{array}{l}\text { Motor } \\
\text { unit }\end{array}$} & \multirow[b]{2}{*}{ Type } & \multirow[b]{2}{*}{$\begin{array}{l}\text { Po } \\
(\mathrm{gm})\end{array}$} & \multirow[b]{2}{*}{ In. ratio } & \multirow[b]{2}{*}{$\begin{array}{l}\text { Terr. size } \\
(\%)\end{array}$} & \multicolumn{2}{|c|}{ Number of fibers } & \multicolumn{2}{|c|}{ Myosin ATPase } & \multicolumn{2}{|c|}{ Fiber diameter } \\
\hline & & & & & Outlined & Depleted & Light & Dark & $\begin{array}{l}\text { Mean } \\
\left(\mu \mathrm{m}^{2}\right)\end{array}$ & $\begin{array}{l}\text { Range } \\
\left(\mu \mathrm{m}^{2}\right)\end{array}$ \\
\hline SOL 1 & $\mathrm{~S}$ & 11.2 & 157 & 41 & 1090 & 49 & 1090 & 0 & 55 & $41-71$ \\
\hline SOL 2 & $\mathrm{~S}$ & 15.6 & 316 & 76 & 1060 & 52 & 1060 & 0 & 59 & $41-87$ \\
\hline SOL 3 & $\mathrm{~S}$ & 13.7 & 177 & 43 & 749 & 53 & 749 & 0 & 50 & $36-73$ \\
\hline TA 1 & $\mathrm{~S}$ & 5.2 & 132 & 8 & 815 & 54 & 159 & 656 & 60 & $19-93$ \\
\hline TA 2 & FR & 9.6 & 188 & 18 & 900 & 49 & 59 & 841 & 69 & $28-120$ \\
\hline TA 3 & FR & 12.7 & 243 & 22 & 982 & 44 & 121 & 861 & 53 & $22-87$ \\
\hline TA 4 & FI & 29.0 & 311 & 12 & 830 & 73 & 164 & 666 & 53 & $21-79$ \\
\hline
\end{tabular}

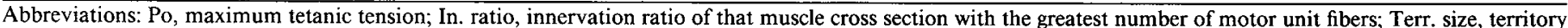

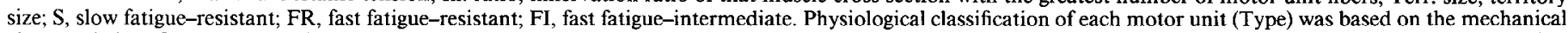

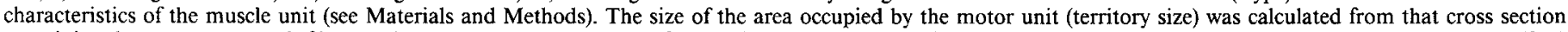

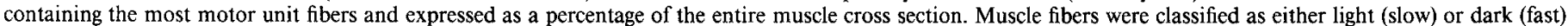
on the basis of their reactions to staining for myosin ATPase and alkaline preincubation.

servations. For example, the expected frequency in category a (Fig. $2 A)$ is

$$
\frac{(a+c) \times(a+b)}{(a+b+c+d)}
$$

The observed values in each category were compared to the expected values for that category, and a chi-square statistic was calculated as the

$$
\sum \frac{(\text { observed }- \text { expected })^{2}}{\text { expected }}
$$

(Dixon and Massey, 1983).

Monte Carlo simulation. In a sample with $n$ motor unit fibers, the number of adjacencies equaled the total number of pairs of depleted fibers that were in contact (Fig. $2 B$ ). In each of the 7 units, the number of adjacencies found in the sample area was determined. The probability of finding that many adjacencies was subsequently determined from a random distribution of adjacencies generated using Monte Carlo simulation techniques.

For each motor unit, a Monte Carlo simulation was performed in which $n$ out of $N$ fibers were chosen at random, where $n$ equaled the number of motor unit fibers, and $N$ the total number of fibers in the sample area. This procedure was repeated 100 times, generating 100 sets of $n$ random numbers, where each random number corresponded to a fiber on the motor unit map. The number of adjacencies in each set of randomly chosen numbers was determined by matching each random number with the corresponding fiber on the sample motor unit map and counting the number of pairs of adjacent fibers. For each motor unit, a frequency distribution of the adjacencies obtained from the simulations was plotted. Using the mean and standard deviation of the frequency distribution of adjacencies generated from the simulation, a $z$ score and $p$ value were calculated for the actual number of adjacencies found within the sample area.

Calculation of a $z$ score and confidence intervals assumes that the populations sampled are normally distributed. Although this assumption may not be true for every distribution generated using the Monte Carlo simulation, the Central Limit Theorem states that if the sample size is large, the distribution of sample means will be approximately normal (Dixon and Massey, 1983). With a sample size of 100 , the confidence intervals and $z$ score will have a precision of \pm 1 .

\section{Distance measurements}

The above measures are limited in that they address only the question of first-order adjacencies and are weak measures of the spatial pattern of motor unit fibers. Two measures that provide more information regarding the spacing of objects in a plane are the distance between a motor unit fiber and its nearest motor unit fiber (nearest-neighbor distance) (Clark and Evans, 1954) and the distance between a motor unit fiber and all other motor unit fibers (interfiber distance) (Ripley, 1981).

Nearest-neighbor distance. For each motor unit fiber in the sample area, the nearest-neighbor distance was defined as the shortest distance between the fiher and another motor unit fiber. Distance measurements were calculated as the distance between the $x, y$ coordinates of the centroids of each fiber:

$$
\sqrt{\left(x_{1}-x_{2}\right)^{2}}+\overline{\left(y_{1}-y_{2}\right)^{2}} \text {. }
$$

When defining the area to be analyzed, the borders or edges of the area were selected to minimize the number of motor unit fibers located on the edge and to ensure that the nearest neighbor of each motor unit fiber was within the defined area. The cumulative distribution of nearestneighbor distances was defined as the total number of motor unit fibers whose nearest neighbor was less than a distance $r$ away.

The statistic with which the actual cumulative distribution was compared was generated using a Monte Carlo simulation. For each sample area of $n$ motor unit fibers and $N$ total fibers, $n$ random fibers were chosen from the sample area and the nearest-neighbor distances and cumulative distribution were calculated as described above. A total of 100 iterations was executed and a $95 \%$ confidence interval generated.

The $95 \%$ confidence interval was defined in the following manner: At each incremental distance, the means and standard deviations of the 100 randomly generated, cumulative nearest-neighbor distances were calculated, and the confidence interval was defined as the mean \pm 1.96 SDs. A continuous curve drawn through each set of points (lower and upper) defined the $95 \%$ confidence interval.

Interfiber distances. The interfiber distances between motor unit fibers were derived in the following manner: For each motor unit fiber in the sample area, the distance between it and every other motor unit fiber was calculated as the distance between the $x, y$ coordinates of the centroids of each fiber. For each pair of motor unit fibers, the distance between them was calculated once; i.e., for $n$ fibers with coordinates $\left(x_{1}, y_{1}\right)$ to $\left(x_{n}, y_{n}\right)$, the distance between all pairs $i, j(i \geq 1$ and $j \leq n)$ was computed for only those pairs $i<j$. Consequently, in a sample of $n$ motor unit fibers, a total of $\left(n^{2}-n\right) / 2$ distances was calculated. The cumulative distribution of interfiber distances was defined as the total number of pairs of fibers with a distance less than $r$.

The statistic with which the actual cumulative distribution was compared was generated using a Monte Carlo simulation, as described for the nearest-neighbor analysis. For each of the 100 iterations, a cumulative distribution of interfiber distances was calculated, and a $95 \%$ confidence interval was generated, as was described for the nearestneighbor analysis.

\section{Results}

To determine whether the spatial arrangement of fibers belonging to a single motor unit departed from random, the distribution of fibers in 3 SOL and 4 TA units was analyzed. Four tests were used to investigate potential local interactions that may occur between motor unit fibers. The adjacency tests were used to determine whether there was some cell-to-cell contact phenomenon that might be reflected in the frequency with which fibers of the same unit were adjacent to one another. The nearestneighbor and interfiber distance analyses provided measures of 


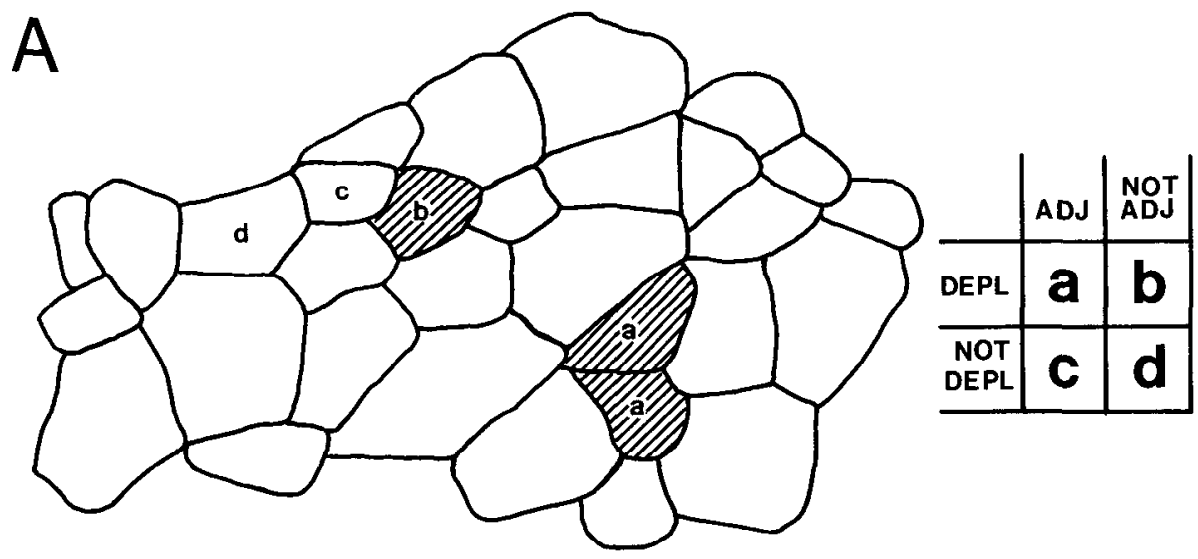

B

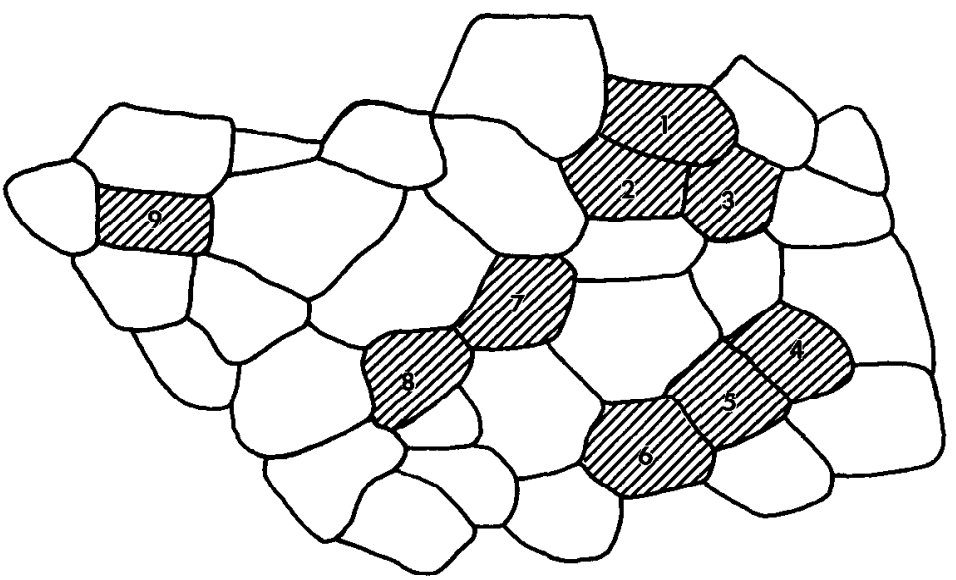

Figure 2. Measurements used in the chi-square $(A)$ and Monte Carlo adjacency $(B)$ analyses. In the the chi-square analysis, fibers were classified into 4 categories according to 2 properties: (1) bclonging to the depleted motor unit ( $D E P L ;$ striped) or not belonging to the depleted motor unit (NOT DEPL; open), and (2) being adjacent $(A D J)$ or not being adjacent $(N O T A D J)$ to a depleted motor unit fiber. In the Monte Carlo adjacency analysis $(B)$, the number of adjacencies was determined by counting the number of pairs of adjacent depleted fibers. For example, among the depleted fibers shown (striped, 1-9), there were 6 adjacencies.

the distance between motor unit fibers and are more useful measures of the local spacing between motor unit fibers.

\section{Chi-square}

The results of the chi-square analyses for the SOL and TA motor units are presented in Figures 3 and 4, respectively. A chi-square value greater than $3.84(p<0.05)$ suggested some interdependence between a fiber belonging to the depleted motor unit and its being adjacent to another fiber of the same unit. The $3 \mathrm{SOL}$ units had chi-square values that were not significantly different from random (Fig. 3). In contrast, 3 of the 4 TA units had significant chi-square values (Fig. 4). Figure 4 shows that TA 2 had fewer adjacencies than expected, whereas TA 3 and TA 4 had more adjacencies than expected.

\section{Monte Carlo simulation}

In this adjacency test, the number of pairs of adjacent motor unit fibers observed in the sample area (actual) was compared to a random distribution of adjacencies generated using Monte Carlo simulation (Figs. 5, 6). The results indicate that the number of pair of adjacencies found in each of the SOL units was not significantly different from random (Fig. 5). Of the 4 TA units, 2 were not significantly different from random (TA 1 and TA 3), one had significantly fewer adjacencies than random (TA 2) and one had more adjacencies than would be expected from a random distribution (TA 4) (Fig. 6). In TA 2, 88\% of the motor unit fibers occurred as singles and the remaining $12 \%$ occurred in groups of 2 . In contrast, only $44 \%$ of the motor unit
A ADJACENT ADJACENT

DEPLETED

NOT-DEPLETED

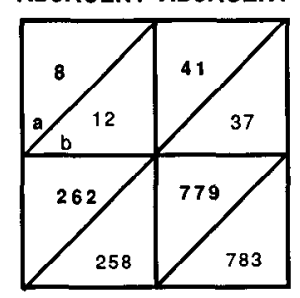

$X^{2}=1.96, \quad p<0.20$
B

ADJACENT ADJACENT

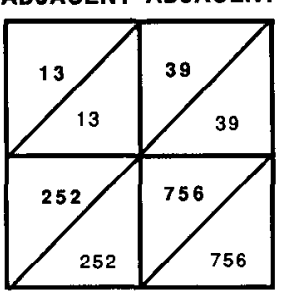

$X^{2}=0.00, p=0$
C

ADJACENT ADJACENT

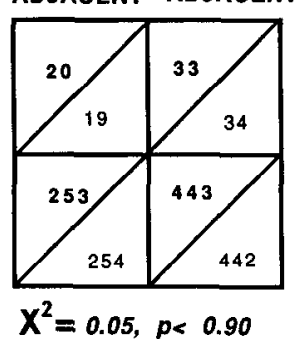

Figure 3. Chi-square analyses for SOL motor units $1(A), 2(B)$, and $3(C) . \Lambda$ chi-square statistic $\left(X^{2}\right)$ was calculated from the observed $(a)$ and expected $(b)$ values. A $p$ value was assigned to each chi-square statistic with a significance level set at $p<0.05$. 


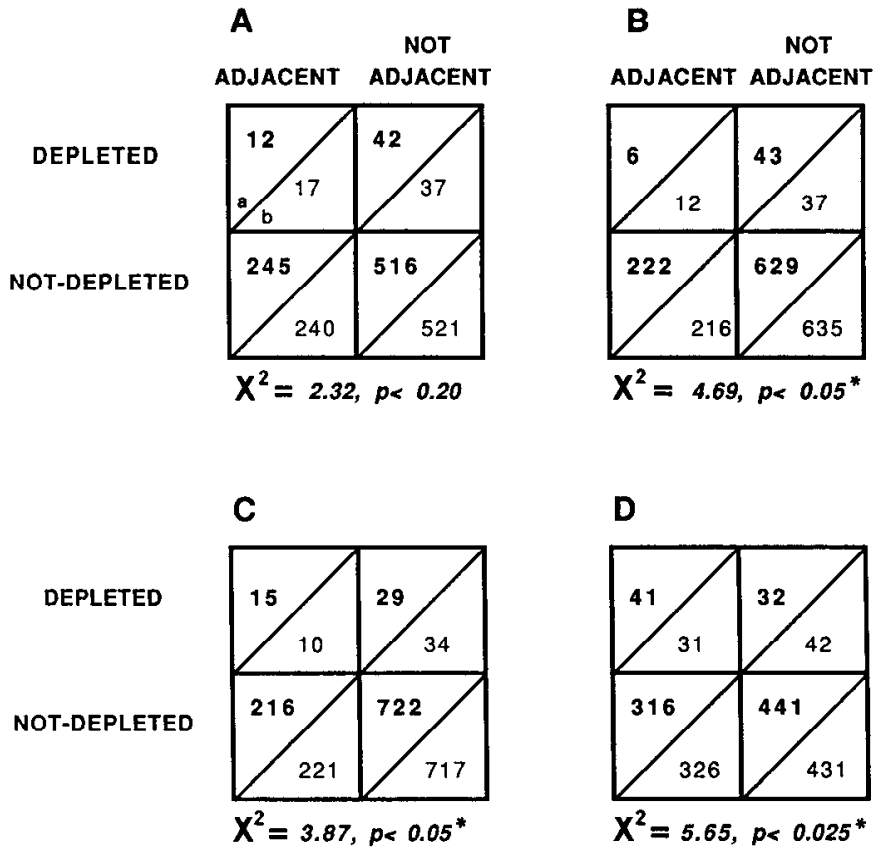

Figure 4. Chi-square analyses for TA motor units $1(A), 2(B), 3(C)$, and $4(D)$. A chi-square statistic $\left(X^{2}\right)$ was calculated from the observed $(a)$ and expected $(b)$ values. A $p$ value was assigned to each chi-square statistic with a significance level set at $p<0.05$.

fibers in TA 4 occurred as singles, while $25 \%$ occurred in groups of $2,25 \%$ in groups of 3 , and $6 \%$ in groups of 5 . For the average motor unit, $71 \pm 14 \%$ of the motor unit fibcrs werc scparated from other motor unit fibers, while $21 \pm 5 \%$ of the motor unit fibers occurred in groups of 2 and only $7 \pm 8 \%$ occurred in groups of 3 . The most fibers of the same unit found in a group of adjacent fibers was 5 , and this occurred only once in TA 4 .

The distribution of adjacencies generated using the Monte Carlo simulation can be influenced by the heterogeneity of fiber cross-sectional area and by fibers located on the edge of the sample area. It is unlikely that heterogeneity of fiber area was a factor in SOL since the range in fiber area was relatively small (2-fold); however, in the TA there was a much greater range in fiber area (10-fold). The error added by variations in fiber area is that larger fibers may have more fibers surrounding their border than do smaller fibers, and, therefore, may have a greater chance of having an adjacency. The relationship between fiber area and the number of fibers in contact with its border was detcrmined for each of the TA units. Spearman rank-correlation coefficients were $0.59,0.59,0.51$, and 0.70 for TA units $1-4$, respectively. These results showed a tendency for the larger fibers to have more fibers in contact with their border than small fibers. However, on the basis of the Monte Carlo simulations, no relationship was observed between the size of a randomly chosen motor unit fiber and its likelihood of having an adjacency to another randomly chosen motor unit fiber.

The effect of choosing a motor unit fiber located on the edge of the sample area is that fibers located on the edge have fewer fibers contacting their border and, therefore, have fewer chances of having an adjacency. To evaluate the effect of choosing fibers on the edge during the simulations, the relationship between the number of edge fibers chosen and the number of adjacencies found in each iteration was determined. For each of the 7 units studied, no relationship $(p<0.05)$ was found. Consequently,

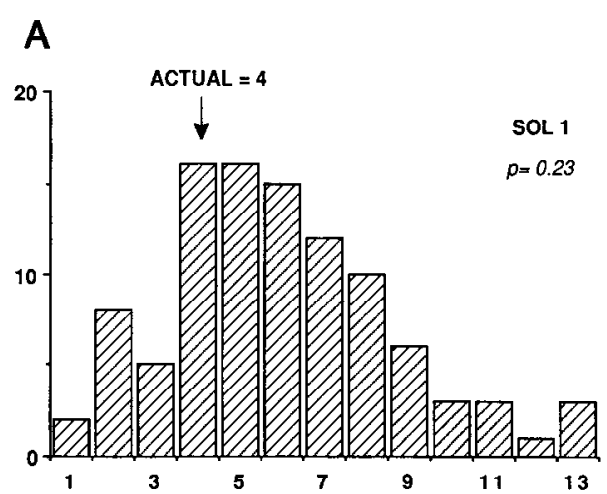

\section{B}
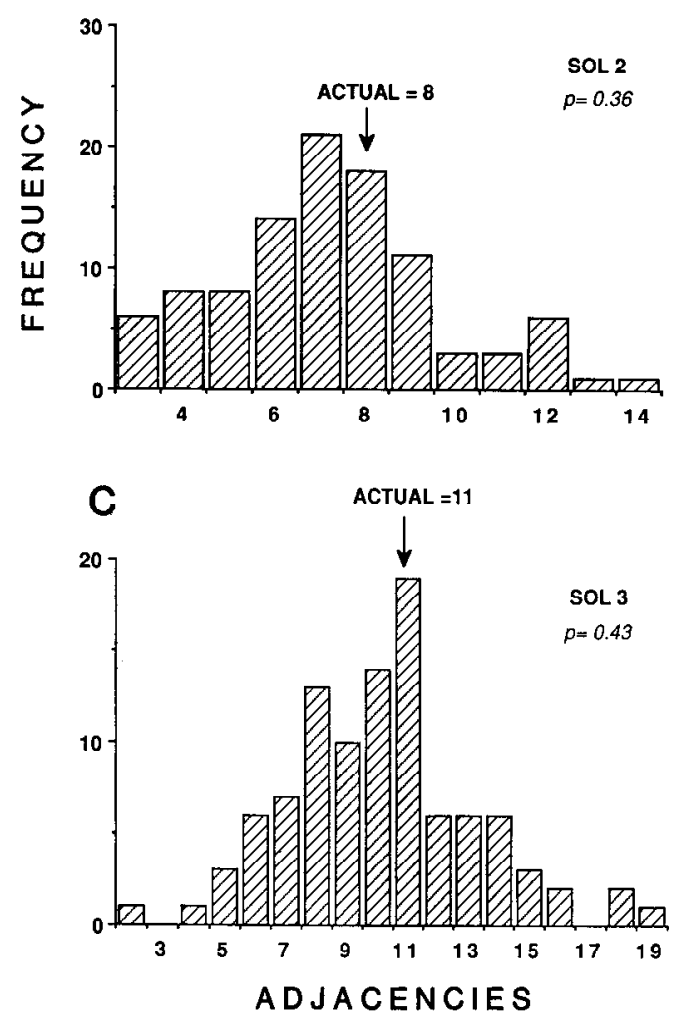

Figure 5. Histograms of the number of adjacencies found in 100 iterations of a Monte Carlo simulation for SOL motor units: $1(A), 2(B)$, and $3(C) . A C T U A L$ refers to the number of adjacencies found in the sample area analyzed from the depleted motor unit. A $p$ value was calculated for the actual number of adjacencies based on the means and standard deviations of the 100 Monte Carlo simulations. Significance was set at $p<0.05$.

fiber area and the number of edge fibers did not appear to bias the random distribution of adjacencies generated using Monte Carlo simulation.

The results of the 2 adjacency tests were consistent. The advantage of the chi-square analysis is that it is a simple, quick test that depends on fiber counts that can be made directly from the histochemical slides. In contrast, Monte Carlo simulations require a detailed spatial map of the motor unit and the surrounding fibers. The advantage of this test over the chi-square test is that it is more robust and requires no assumptions regarding the expected number of adjacencies. 


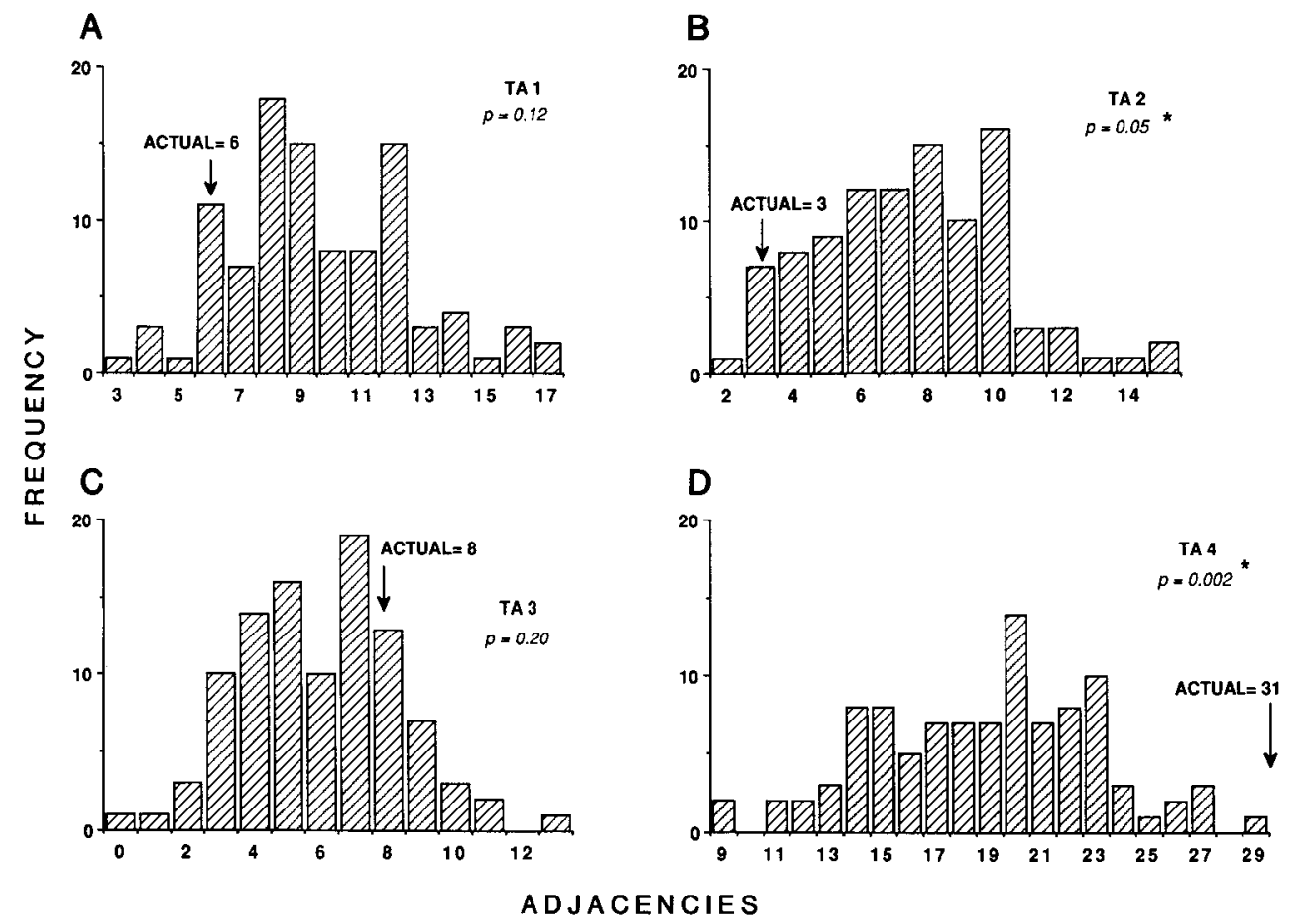

Figure 6. Histograms of the number of adjacencies found in 100 iterations of a Monte Carlo simulation for TA motor units $1(A), 2(B)$, and $3(C)$, and $4(D) . A C T U A L$ refers to the number of adjacencies found in the sample area analyzed from the depleted motor unit. A $p$ value was calculated for the actual number of adjacencies based on the means and standard deviations of the 100 Monte Carlo simulations. Significance was set at $p<0.05$.

\section{Nearest-neighbor distances}

The cumulative distribution of the actual nearest-neighbor distances and the $95 \%$ confidence interval for a random distribution are plotted for the SOL (Fig. 7) and the TA (Fig. 8) units. The distribution of nearest-neighbor distances was considered to be significantly different from random if it fell outside of the envelope defined by the $95 \%$ confidence interval.

The 3 SOL units studied had nearest-neighbor distributions that remained within the $95 \%$ confidence intervals (Fig. 7), suggesting that the local spacing between motor unit fibers is random. It should be noted, however, that the farthest distances between nearest-neighbor motor unit fibers were 282, 299, and $271 \mu \mathrm{m}$ in SOL $1-3$, respectively. These distances correspond to a maximum separation between nearest neighbors of approximately 5 fibers.

Figure 8 shows that of the 4 TA units studied, 2 (TA 1 and 2) deviated from random and 2 (TA 3 and 4) remained within the $95 \%$ confidence interval. The distribution of TA 1 deviated from the lower limit at distances of $134-190 \mu \mathrm{m}$, which corresponds to an avoidance of distances equal to approximately 2-3 fibers. In unit TA 2, the distribution deviated from the lower limit at distances ranging from 72 to $274 \mu \mathrm{m}$, which corresponds to an avoidance of distances equal to approximately 1-4 fibers. In both these muscles, these results suggest a local spreading or dispersion of motor unit fibers.

It is interesting to note that, although both TA 1 and TA 2 showed evidence of spreading, TA 1 had a random distribution of adjacencies, whereas, TA 2 had fewer adjacencies among motor unit fibers than expected from a random process. These differences in the number of adjacencies are also apparent in the results of the nearest-neighbor analysis. Note that in TA 1 (Fig. $8 A$ ), the actual distribution is within the $95 \%$ confidence interval at a distance of $60 \mu \mathrm{m}$, which approximates the average diameter of muscle fibers in that sample area (Table 1). In contrast, the actual distribution of TA 2 (Fig. $8 B$ ) deviates from random at a distance equivalent to the average diameter of fibers in that sample area (Table 1). In all but one unit, the results of the nearest-neighbor analyses were consistent with the results of the adjacency tests. In TA 4, the adjacency tests indicated that there were more adjacencies than expected from a random process. The nearest-neighbor analysis, however, did not deviate from random at distances equal to the average fiber diameter in that unit (Table 1.). The discrepancy between these tests is likely related to the fact that, in the area sampled for TA 4 , the fibers belonging to the unit were considerably larger than those not belonging to the unit. Since the motor unit fibers were larger than the non-motor unit fibers, the distances between adjacent fibers in the motor unit were larger than the average distance between adjacent fibers in the simulations of TA 4 . Note that although the nearest-neighbor distribution of TA 4 (Fig. 8D) did not deviate from random, there was a large increase in added fibers of between 50 and $60 \mu \mathrm{m}$, which corresponds to the number of adjacencies found in the unit.

In the initial random simulations, fiber type, i.e., fast or slow, was ignored. To account for the possibility that fiber type may be specified prior to innervation and that motoneurons may be selective to specific fiber types, a series of simulations were performed in which only those fibers of the same fiber type as the depleted motor unit were sampled. That is, if a unit was fast, only fast fibers would be randomly chosen from the sample area. These simulations were made only for the TA units, since the SOL is a homogeneous slow muscle. The results of these simulations were similar to those with the initial simulations, which ignored fiber type differences.

\section{Interfiber distances}

A description of spatial patterns hased solely on nearest-neighbor distances is not really complete, for it disregards the other spatial relations that exist between fibers. For example, the nearest-neighbor measure could not differentiate between a population of motor unit fibers congregated in a small percentage of 
A
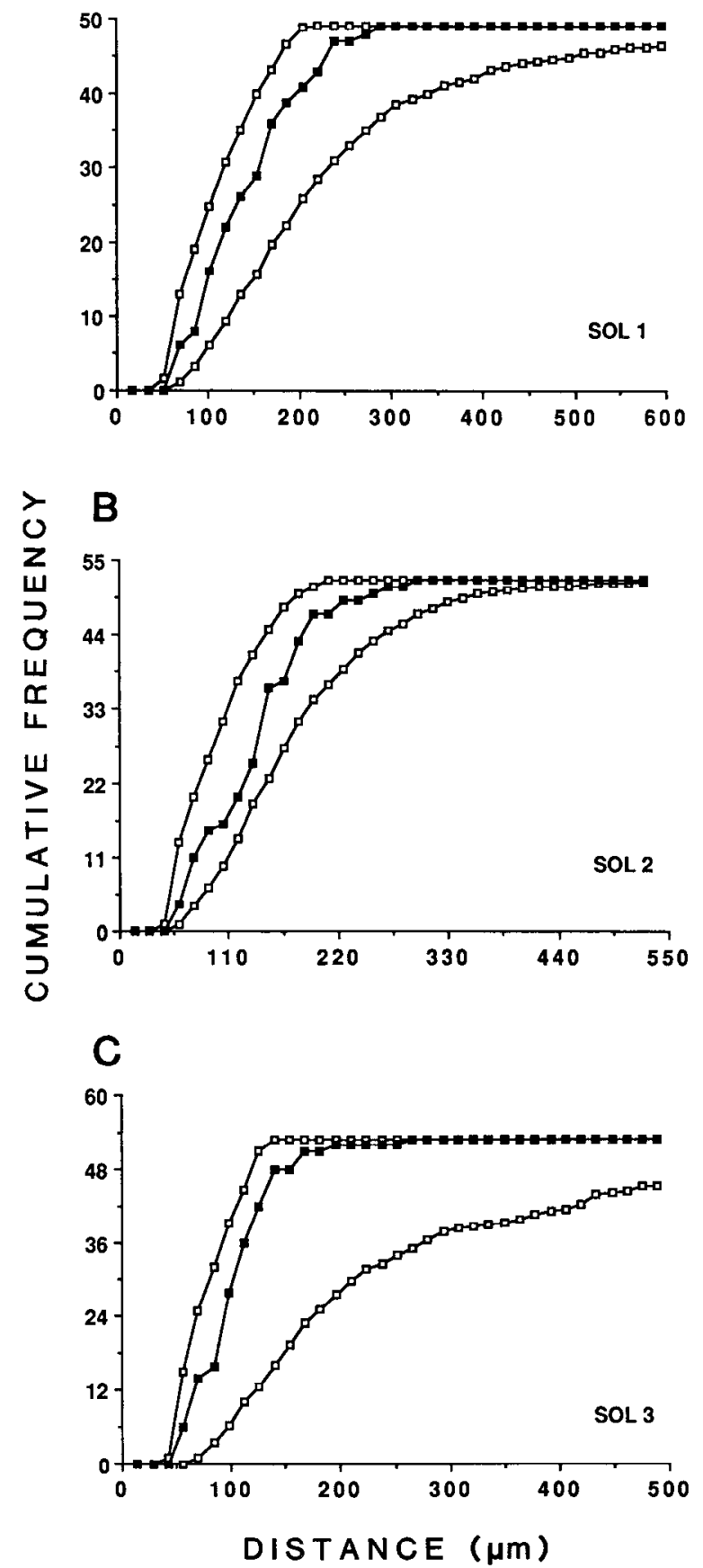

Figure 7. Total number of nearest-neighbor distances less than some incremental distance (cumulative frequency) versus the distance between motor unit fibers for SOL units $1(A), 2(B)$, and $3(C)$. The actual distribution is represented by the solid squares and the $95 \%$ confidence interval, determined from the simulations, by the open squares.

the sample region and a population of fibers consisting of pairs of motor unit fibers distributed throughout the sample region. To obtain a more complete measure of the spacing of motor unit fibers, the distances betwecn onc motor unit fibcr and all others in the sample area were calculated.

Figures 9 and 10 summarize the cumulative distributions of the interfiber distances measured between all motor unit fibers in the sample areas taken from 3 SOL and 4 TA units, respec- tively. Of the 3 SOL units studied (Fig. 9), only SOL 1 had a distribution that deviated from the $95 \%$ confidence interval. In this unit, the distribution departed from the upper limit at a distance of $149 \mu \mathrm{m}$. Calculation of the number of pairs added at each interval revealed that the most significant increase in the number of pairs of fibers was between 200 and $1000 \mu \mathrm{m}$. Although the distributions of both SOL 2 and SOL 3 remained within the $95 \%$ confidence interval, there were intervals where they approached either the upper or lower limits of the confidence interval. For example, in SOL 2 there was a significant decrease in the number of pairs with interfiber distances between 1035 and $1350 \mu \mathrm{m}$, while in SOL 3 there was a significant increase in the number of pairs separated by distances between 344 and $473 \mu \mathrm{m}$.

Figure 10 shows that, of the 4 TA units studied, only one unit (TA 4) had a distribution that deviated from random. In TA 4 (Fig. $10 D$ ), the distribution deviated from the upper limit at a distance of $86 \mu \mathrm{m}$. Closer inspection of the curves revealed that the stecpest risc in the cumulative distribution occurred between 465 and $899 \mu \mathrm{m}$.

In general, the cumulative distributions of interfiber distances showed a tendency to deviate more towards the upper limit than the lower limit. This finding might suggest a limitation in the amount of spread or dispersion of fibers belonging to a motor unit. For example, the furthest separations between any 2 motor unit fibers in those areas sampled ranged from 35 to 49 fibers.

\section{Discussion}

The distribution pattern of muscle fibers belonging to a motor unit reflects the net result of the control exerted by mechanisms involved in nerve-muscle connectivity. A high degree of matching has been shown between discrete motoneuron pools and muscles, suggesting that motoneurons do not innervate the limb in a random manner (for review, see Landmesser, 1980, and Bennett, 1983). The pathway taken by a ncrve to a specific muscle appears to be discrete, with few projection errors occurring during normal development (Landmesser, 1980). By contrast, little is known about the specificity of the branching pattern of a motor axon or the spatial arrangement of those muscle fibers innervated by a single motoneuron.

At birth, muscle fibers are innervated by several motoneurons. Subsequently, after a periud of synapse elimination, the number of muscle fibers innervated by each motoneuron is reduced and each muscle fiber becomes innervated by only one motoneuron (for review, see Van Essen, 1982, and Thompson, 1985). The elimination of excess motoneuron contacts could be a random process; however, a process based solely on random elimination of synapses would likely result in some fibers' becoming denervated. Synapse elimination appears to be influenced by at least 2 processes: (1) competition between different axons for the same muscle fiber, and (2) an inherent tendency for motoneurons to retract synapses (Van Essen, 1982; Thompson, 1985). What factor(s) the axons are competing for and what determines which axons will survive is unknown. It has been reported that synapse elimination does not appear to affect which motoneurons innervate which muscles (Brown et al., 1976; Landmesser, 1984). However, synapse elimination could have some role in determining the topographical mapping of motoneurons onto the fibers within the muscle and could alter the spatial distribution of fibers belonging to a single motor unit at several levels. Locally, a fiber could be eliminated because it is in direct contact with another fiber belonging to the same motor 


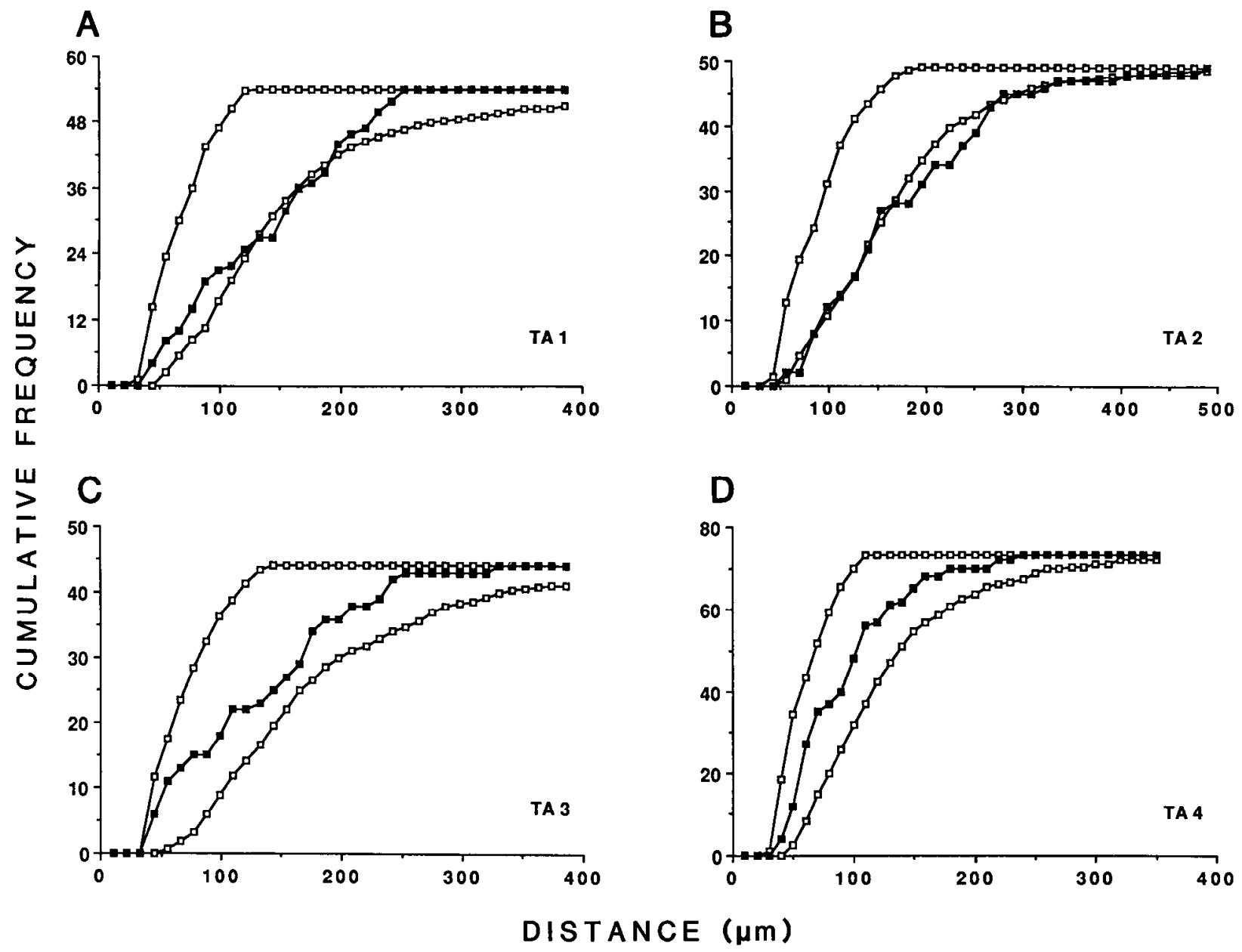

Figure 8. Total number of nearest-neighbor distances less than some incremental distance (cumulative frequency) versus the distance between motor unit fibers for TA units $1(A), 2(B), 3(C)$, and $4(D)$. The actual distribution is represented by the solid squares and the $95 \%$ confidence interval, determined from the simulations, by the open squares.

unit. On a larger scale, synapse elimination could restrict the absolute size of the territory innervated by a motoneuron. Consequently, at one level of organization, one might expect to find dispersion of motor unit fibers, while at another level one might expect to find a grouping of motor unit fibers. Because several mechanisms may be influencing the motor unit distribution, the spatial patterns at several different levels are of interest. The analyses performed in this paper were designed to investigate the local organization of the motor unit, i.e., the spatial patterns that exist in a selected region of the motor unit as opposed to patterns that exist over the entire motor unit territory.

\section{Distribution of motor unit fibers}

The adjacency tests addressed the question of whether there is some mechanism that prevents fibers of the same motor unit from being adjacent to one another. Brandstater and Lambert (1973) described the distribution of motor unit fibers in 23 TA units of the rat as being "diffusely scattered throughout the motor unit territory, with little or no tendency for grouping." In addition, they found no greater tendency for grouping or dispersion in the motor unit fibers of these units than in units that were simulated by randomly choosing squares on graph paper that were designed to represent the territory of a motor unit. In contrast, Willison (1980) concluded that the arrange- ment of muscle fibers within a unit was ordered or "non-random," in that there were fewer adjacent motor unit fibers than expected from random. His argument was based on a comparison of the actual number of adjacencies found in 3 depleted rat units to a model that included only 19 cells in an assumed hexagonal arrangement of muscle fibers. Using this model, 19 "colors" were chosen at random from a pool of "colors" (1 through $N$ ), where $N$ equaled the number of motoneurons innervating the motor unit territory under study. The number of motoneurons innervating a specific territory was determined by the percentage of the cross section occupied by the unit and the total number of axons innervating the muscle. For example, if a unit occupied $10 \%$ of the cross section, then it could be predicted that $10 \%$ of the motoneurons innervating the muscle would innervate the area occupied by that motor unit. One limitation to this model is that the size of the model motor unit is small relative to the size of these motor units studied and, consequently, an artifically large number of adjacencies will be found in the simulations. This approach results in a strong bias toward finding fewer adjacencies in the actual data than would be expected from a random distribution. An additional limitation is that the calculation of the number of motoneurons innervating a given territory does not account for compartmentalization within a muscle or for overlapping and unequal-sized 

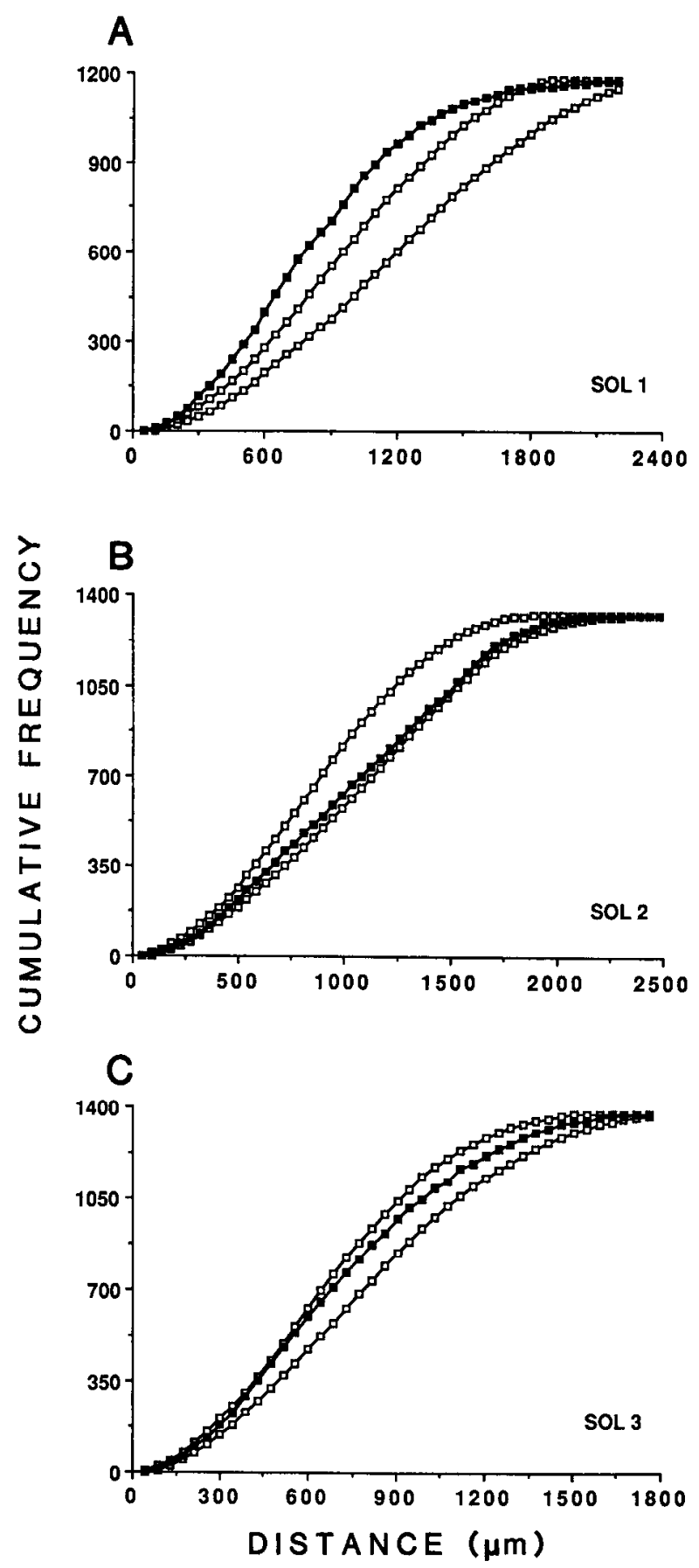

Figure 9. Total number of interfiber distances less than some incremental distance (cumulative frequency) versus the distance between motor unit fibers for SOL units, $1(A), 2(B)$, and $3(C)$. The actual distribution is represented by the solid squares and the $95 \%$ confidence interval, determined from the simulations, by the open squares.

motor unit territories. For example, in the TA of the cat there are 2 compartments: the anterior compartment innervated by approximately $72 \%$ of the motoneurons in the TA motor pool, and the posterior compartment, innervated by the remaining 18\% (Iliya and Dum, 1984). Consequently, using Willison's (1980) assumptions, the number of motoneurons in this muscle that innervate a specific area would be overestimated, especially if the motor unit resided in the posterior compartment.

In the present study, a random distribution of adjacencies was simulated for each motor unit. The simulation involved determining what a random distribution would be, given that a known number of fibers within an outlined region had to be motor unit fibers. Using this approach, it was not necessary to make any assumptions regarding the number of motoneurons innervating a given area or the arrangement of muscle fibers within the sample. This method, therefore, produced a test statistic based on fewer assumptions with which to compare the actual and expected data. Results from the analysis of $3 \mathrm{SOL}$ and 4 TA motor units suggest that there was no forceful process that prevented or encouraged motor unit fibers to be in contact with one another.

On the basis of the adjacency tests used in this study, there were no influences that tended to prevent the direct contact of motor unit fibers. These findings, however, do not preclude the presence of some influence that could function to maintain motor unit fibers at some preferred distance apart. Such a factor might be elucidated by analyzing the distance between nearest neighbors of motor unit fibers or the interfiber distances between all motor unit fibers.

The results of the nearest-neighbor analysis do not give conclusive evidence for a mechanism that spreads motor unit fibers at short distances. Of the 7 units studied, only 2 (TA 1 and 2) showed a level of dispersion between motor unit fibers that was significantly greater than random. It is interesting to note, however, that a motor unit fiber was never separated from its nearest motor unit fiber by more than 7 fibers.

One of the TA units that did show a significant spreading effect was the slow unit (TA 1). It has been noted (James, 1971) that type I (slow) fibers in a mixed muscle appear to have a distribution that is more regular than random. To address the possibility that the "spreading" found in TA 1 was a consequence of the distribution of slow fibers, a nearest-neighbor calculation and simulation was done on the slow fibers contained within the motor unit territory of TA 1 (Table 1). For each slow fiber in the sample area, the nearest-neighbor distance was defined as the shortest distance between it and another slow fiber. Consequently, 100 iterations of a Monte Carlo simulation were done in which the number of random fibers chosen was equal to the number of slow fibers in the sample area. The results showed that the slow fibers within the sample region had a random distribution, i.e., fell within the $95 \%$ confidence interval. This suggests that the spreading seen in TA 1 did not appear to be due to some type-related phenomenon. The nearest-neighbor distributions of the slow fibers were calculated for the remaining TA units, and, in each muscle, the slow fibers were randomly distributed within the sample region. A random distribution of type I (slow) fibers also has been reported by Lexell et al. (1983).

Calculation of all interfiber distances between motor unit fibers revealed a tendency for the motor unit to be more "grouped" or "restricted" than dispersed. This result might have been expected, given that histochemical mapping of glycogen-depleted motor units has shown that motor unit fibers are not usually distributed across the entire muscle cross section, but are somewhat localized (Edstrom and Kugelberg, 1968; Bodine et al., 1987). This observation suggests that some mechanism exists whereby the territory of a motor unit is restricted. Brown and Booth (1983) reported that the territory over which rat gluteus motor units are distributed decreases after the period of synapse elimination. A comparable localization of the innervation pattern has been shown to develop in the frog gluteus (Bennett and 


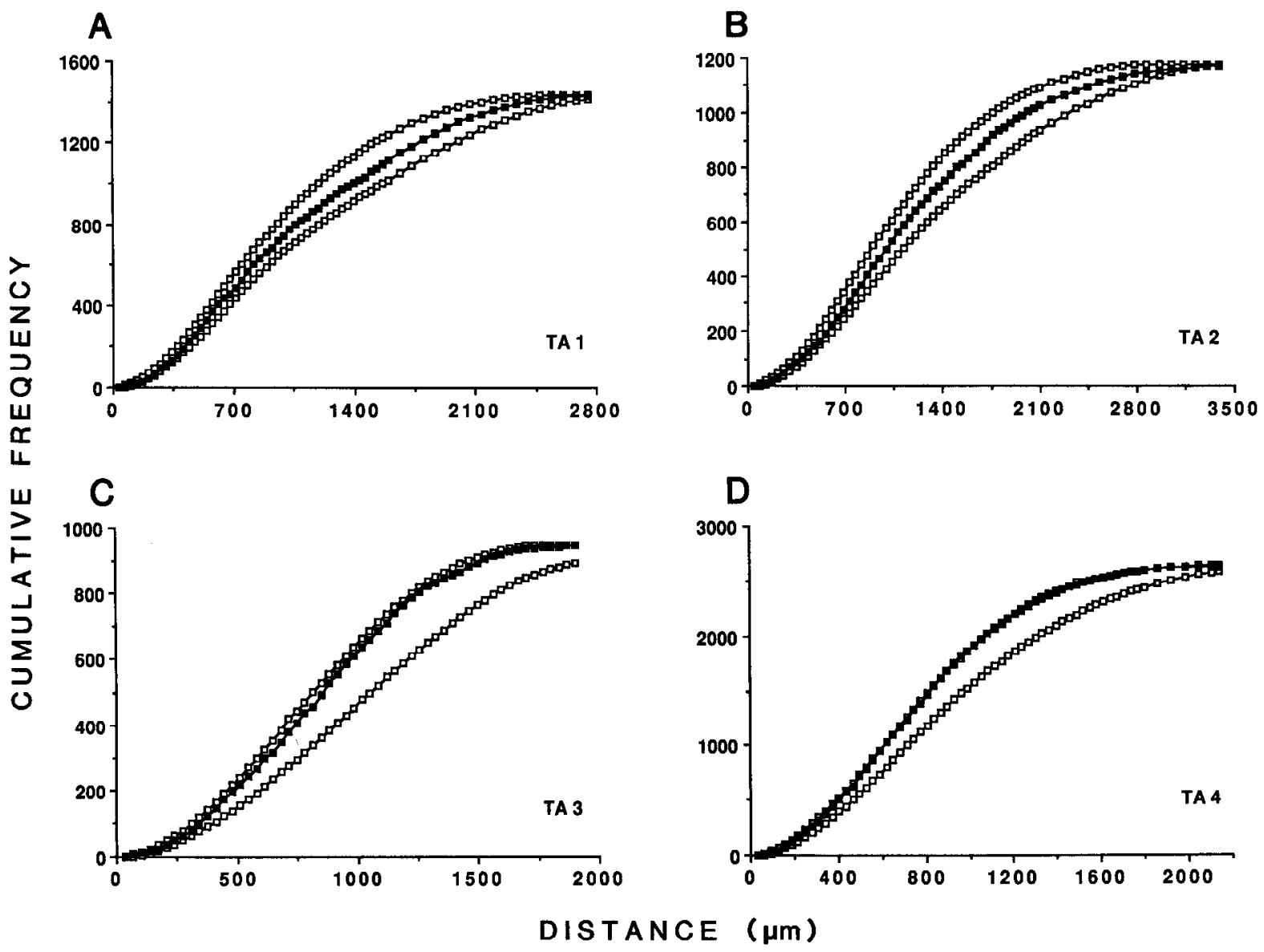

Figure 10. Total number of interfiber distances less than some incremental distance (cumulative frequency) versus the distance between motor unit fibers for TA units, $1(A), 2(B), 3(C)$, and $4(D)$. The actual distribution is represented by the solid squares and the $95 \%$ confidence interval, determined from the simulations, by the open squares.

Lavidis, 1982) and rat gastrocnemius (Bennett and Lavidis, 1984).

The analyses presented in this paper were used to quantify the local or short-range spatial patterns of motor unit fibers in the soleus and tibialis anterior muscles of the cat. Although there were apparently no strong fiber-fiber interactions operating at short distances that tended to keep fibers of the same unit spread apart, in the 7 units studies we found some support for a mechanism that restricted the absolute distribution and territory of motor unit fibers.

\section{References}

Bennett, M. R. (1983) Development of ncuromuscular synapses. Physiol. Rev. 63: 915-1048.

Bennett, M. R., and N. A. Lavidis (1982) Development of the topographical projection of motor neurons to amphibian muscle accompanies motor neuron death. Dev. Brain Res. 2: 448-452.

Bennett, M. R., and N. A. Lavidis (1984) Development of the topographical projection of motor neurons to a rat muscle accompanies loss of polyneuronal innervation. J. Neurosci. 9: 2204-2212.

Bodine, S. C., R. R. Roy, E. Eldred, and V. R. Edgerton (1987) Maximal force as a function of anatomical features of motor units in the cat tibialis anterior. J. Neurophysiol. 57: 1730-1745.

Brandstater, M. E., and E. H. Lambert (1973) Motor unit anatomy. In New Developments in EMG and Clinical Neurophysiology, J. E. Desmedt, ed., pp. 14-22, S. Karger, Basel.

Brown, M. C., and C. M. Booth (1983) Postnatal development of the adult pattern of motor axon distribution in rat muscle. Nature 304: $741-742$.
Brown, M. C., J. K. S. Jansen, and D. C. Van Essen (1976) Polyneuronal inncrvation of skclctal muscle in new-born rats and its climination during maturation. J. Physiol. (Lond.) 261: 387-422.

Buchthal, F., C. Guld, and P. Rosenfalck (1957) Multielectrode study of the territory of a motor unit. Acta Physiol. Scand. 38: 331-354.

Castleman, K. R., L. A. Chui, T. P. Martin, and V. R. Edgerton (1984) Quantitative muscle biopsy analysis. In Computer Enhanced Imagery in Cytopathology. Monographs in Clinical Cytology, S. D. Greenberg, ed., pp. 101-116, S. Karger, Basel.

Clark, P. J., and F. C. Evans (1954) Distance to nearest neighbors as a measure of spatial relationships in populations. Ecology 35: 445453.

Cope, T. C., S. C. Bodine, M. Fournier, and V. R. Edgerton (1986) Soleus motor units in chronic spinal transected cats: Physiological and morphological alterations. J. Neurophysiol. 55: 1202-1220.

Dennis, M. J., L. Ziskind-Conhaim, and A. J. Harris (1981) Development of neuromuscular junctions in rat embryos. Dev. Biol. 81 : 266-279.

Dixon, W. J., and F. J. Massey (1983) Introduction to Statistical Analysis, McGraw-Hill, New York.

Donselaar, Y., D. Kernell, O. Eerbeek, and B. A. Verhey (1985) Somatotopic relations between spinal motoneurones and muscle fibers of the cat's musculus peroneus longus. Brain Res. 335: 81-88.

Edstrom, L., and E. Kugelberg (1968) Histochemical composition, distribution of fibres and fatigability of single motor units. J. Neurol. Neurosurg. Psychiatry 31: 424-433.

English, A. W., and O. I. Weeks (1984) Compartmentalization of single muscle units in cat lateral gastrocnemius. Exp. Brain Res. 56: 361368.

Garfinkcl, A., S. C. Bodinc, R. R. Roy, and V. R. Edgcrton (1986) Spatial distribution of single motor unit fibers in the cat soleus and tibialis anterior muscles. Soc. Neurosci. Abstr. 12: 319. 
Gath, I., and R. Shenhav (1985) Probabilistic model of the spatial distribution of muscle fibres in human muscles. Biol. Cybern. 53: 7378 .

Iliya, A. R., and R. P. Dum (1984) Somatotopic relations between the motor nucleus and its innervated muscle fibers in the cat tibialis anterior. Exp. Neurol. 86: 272-292.

James, N. T. (1971) A geometrical probability study of type I muscle fibres in the rabbit and guinea pig. J. Neurol. Sci. 14: 381-387.

Kugelberg, E. (1973) Properties of the rat hind-limb motor units. In New Developments in EMG and Clinical Neurophysiology, J. E. Desmedt, ed., pp. 2-13, S. Karger, Basel.

Landmesser, L. T. (1980) The generation of neuromuscular specificity. Ann. Rev. Neurosci. 3: 279-302.

Landmesser, L. T. (1984) The development of specific motor pathways in the chick embryo. Trends Neurosci. $7: 336-339$.

Laskowski, M. B., and J. R. Sanes (1987) Topographic mapping of motor pools onto skeletal muscles. J. Neurosci. 7: 252-260.

Lexell, J., D. Downham, and M. Sjostrom (1983) Distribution of different fibre types in human skeletal muscles. J. Neurol. Sci. 61: 301-314.

Martin, T. A., A. C. Vailas, J. B. Durivage, V. R. Edgerton, and K. R. Castleman (1985) Quantitative histochemical determination of muscle enzymes: Biochemical verification. J. Histochem. Cytochem. 33: 1053-1059.

McLennan, I. S. (1983) The development of the pattern of innervation on chicken hindlimb muscles: Evidence for specification of nervemuscle connections. Dev. Biol. 97: 229-238.
Miller-Larsson, A. (1980) A modcl of spatial distribution of muscle fibres of a motor unit in normal human limb muscles. Electromyogr. Clin. Neurophysiol. 20: 281-298.

Nwoye, L., W. F. H. M. Mommaerts, D. R. Simpson, K. Seraydarian, and M. Marusich (1982) Evidence for direct action of thyroid hormone in specifying muscle properties. Am. J. Physiol. 242: R401R408.

Pearse, A. G. E. (1961) Histochemistry-Theoretical and Applied, Little, Brown, Boston.

Purves, D., and J. W. Lichtman (1984) Principles of Neural Development, pp. 271-300, Sinauer, Sunderland, MA.

Ripley, B. D. (1981) Spatial Statistics, pp. 144-190, Wiley, New York.

Thompson, W. J. (1985) Activity and synapse elimination at the neuromuscular junction. Cell. Mol. Neurobiol. 5: 167-182.

Thompson, W. J., L. A. Sutton, and D. A. Riley (1984) Fibre typc composition of single motor units during synapse elimination in neonatal rat soleus muscle. Nature 309: 709-711.

Van Essen, D. C. (1982) Neuromuscular synapse elimination. In Neuronal Development, N. C. Spitzer, ed., pp. 333-376, Plenum, New York.

Weeks, O. I., and A. W. English (1985) Compartmentalization of the cat lateral gastrocnemius motor nucleus. J. Comp. Neurol. 235: 255267.

Willison, R. G. (1980) Arrangement of muscle fibers of a single motor unit in mammalian muscles. Muscle Nerve 3: 360-361. 